\title{
Significance of Transportation Network Models in Emergency Planning of Urban Cities
}

\author{
Tanka Nath Dhamala ${ }^{1}$ and Urmila Pyakurel ${ }^{2}$ \\ Central Department of Mathematics, Tribhuvan University, Kathmandu, Nepal \\ Email: ${ }^{1}$ amb.dhamala@daadindia.org, ${ }^{2}$ urmilapyakurel@gmail.com
}

\begin{abstract}
Transportation network models within the framework of operations research have been established as one of the emerging field of research frontiers in today's very disastrous life and dense traffic system. The discrete as well as continuous flow over time models have been widely considered by social, engineering and applied scientists with wide varieties of real-life problems, like rush hour traffic and traffic management at the time of any disaster. Among the PPRR classification issues, the concentration will be given within the planning approach with transportation networks for urban cities.
\end{abstract}

We present the models and solution strategies of the transportation networks that are dynamic in nature and directly apply in rush hour traffic or human-made or natural disasters in the urban cities. The difficulty levels of the varieties of algorithms, efficiencies of their solution software and the significance of the obtained solutions for the betterment of modern city plan are discussed with wide spectrum of model diversity. Among many others, we illustrate, also supporting with a case study, the impact of recent Nepal Earthquake 2015 and the meaningfulness of effective evacuation planning for saving the property and people in urban city like, Kathmandu, valley.

The high performance of the technique of lane reversals in transportation or evacuation networks which has already been adopted a lot in practice, but has been analytically studied only recently are focused in this paper. Adopted this dynamic transportation strategy, the flow in the transportation can be doubled, the time can be saved significantly and many transportation arcs can be utilized for logistic supports or for emergency vehicles whenever necessary. Improved results are presented and the importance of integrated models dependent on time and vehicles is explored. The optimal solution thus obtained with contraflow, logistic supports and facility location at appropriate positions of transportation network proves the significance of these models in emergency planning.

Key words: network optimization, transportation network, evacuation planning, contraflow, heuristics.

2010 Mathematics Subject Classification. Primary: 90B10, 90C27, 68Q25; Secondary: 90B06, 90B20. 


\section{Introduction}

The most horrible disasters have been motivating research in emergency evacuation, for example, see the reviews (Hamacher and Tjandra, 2002; Pardalos and Arulselvan, 2009; Pel et al. 2012; Yusoff et al. 2008; Dhamala, 2015). A network models an evacuation region (building), where the intersections of streets in a region (rooms in a building) represent the nodes and connections between these parts correspond to edges. There are capacity constraints on nodes and edges, and given evacuation times on edges. The arc flow capacity represents the amount of travellers allowed to pass through the arc per unit time and the arc evacuation time is the time restricted to travel from one node to the other. The models with real evacuation time (travel time and response time) and continuous time settings yield more accuracy. But the integrated models impose high complexity. Therefore, large-scale planning models only consider the travel time and constant parameters with discrete settings for a lower bound result of the realistic model.

The models and solution methods depend on factors like problem size, behavioural and organizational situations, transportation modes, traffic capacity, time dependency and evacuation objectives, (Hamacher and Tjandra, 2002). Pardalos and Arulselvan (2009) classify the models and solution methods based on the computational performance, scalability, extensibility and reliability. The models can be classified into macroscopic and microscopic. The former mostly deal with optimization methods, whereas the latter is mainly based on simulation experiments which mostly rely on cellular automata. The lane reversals are the best among heuristics. Pel et al. (2012) review mathematical and traffic simulation models dealing with behavioural limitations of evacuees. Yusoff et al. (2008) give a survey on the macroscopic models considering the number of evacuees and the evacuation time as most important objectives for the application to real-world evacuation. Dhamala (2015) reviews the discrete evacuation problems in detail.

Nepal Earthquake of magnitude 7.8 Richter scale that happened on 25 April 2015 and two powerful aftershocks killed at least 8,891 people, injured 22,302 people, fully damaged 602,567 and partially damaged 284,479 private homes, 2,687 government offices are fully damaged and 3,776 are partially damaged. After any kind of disasters, the problem of saving evacuees and normalizing the situation is challenging. An effective evacuation solution has been lacked without a prior evacuation planning. A case study carried out before this disaster, but is still applicable, has been given in Section 7.

The fundamental models of network flows over time are reviewed in Section 2. We study the evacuation problems (maximum (MDF), quickest time (QF), lexicographically maximum (LMDF), and earliest arrival (EAF) in Section 3. Section 4 deals with the contraflow solutions and a compact study on the integrated approach of transit-based and auto-based evacuation. Section 5 highlights the facility location models and solution approaches. The simulation approaches and existing software are reviewed in Section 6. We state several exact algorithms and heuristics and notable simulation software characteristics in brief. The simplest models with single (source, sink) to complex models with multiterminal are covered. This work mostly integrates several efforts made during a long time span. The paper concludes with a list of findings and the scope of further research exposures. 


\section{The Flow Networks}

Let $\mathcal{N}=(V, A)$ be a directed network with $n$ nodes and $m$ arcs, and let $A(v)$ and $B(v)$ be the sets of arcs from and to node $v$, respectively, where $B(s)=A(t)=\varnothing$ for the single-source $s$ and the single-sink $t$. The capacity, travel time and cost per unit flow on the arc $e$ are denoted by $b_{A}(e)$, $\tau(e)$, and $c(e)$, respectively. The capacity and initial occupancy, if any, at node $v$, are denoted by $b_{V}(v)$ and $o(v)$, respectively. Though complicated, the total number of evacuees is estimated by $p$. The associated parameters may be flow and/or time dependent, but we assume them to be constants, if not stated otherwise. Sections 3.3 and 3.2 deal with the general cases of parameters. The permissible evacuation egress time horizon is assumed to be $T$.

\subsection{Discrete model}

Let the given time be discretized as $\mathbf{T}=\{0,1, \ldots, T\}$. A discrete dynamic $s-t$ flow $x_{d}: A \times$ $\mathbf{T} \rightarrow \mathrm{R}^{+}$with given $T$ is represented by the flow conservation and capacity constraints (1-3), where $x_{d}(e, \theta)$ gives the number of evacuees that start moving along $e$ at time $\theta$ and reach its end after time $\tau(e)$. The Constraint (2) permits holding the flow at intermediante nodes.

$$
\begin{array}{ll}
\sum_{\sigma=\tau(e)}^{T} \sum_{e \in B(v)} x_{d}(e, \sigma-\tau(e))-\sum_{\sigma=0}^{T} \sum_{e \in A(v)} x_{d}(e, \sigma)=0, & \forall v \notin\{s, t\} \\
\sum_{\substack{\sigma=\tau(e) \\
0 \leq x_{d}(e, \theta) \leq b_{A}(e, \theta),}} x_{d}(e, \sigma-\tau(e))-\sum_{\substack{\sigma=0 \\
0}} \sum_{e \in A(v)} x_{d}(e, \sigma) \geq 0, & \forall v \notin\{s, t\}, \forall \theta \in \boldsymbol{T} \\
0 e \in A, \forall \theta \in \boldsymbol{T} &
\end{array}
$$

The objective function is represented by (4) as the total flow entering the sink (leaving the source).

$v_{\mathrm{T}} x_{d}=\sum_{\sigma=0}^{\theta} \sum_{e \in A(s)} x_{d}(e, \sigma)=\sum_{\sigma=\tau(e)}^{\theta} \sum_{e \in B(t)} x_{d}(e, \sigma-\tau(e))$

We refer to the pioneer work of (Ford and Fulkerson, 1958) for the modeling of discrete dynamic flow problems.

The MDF problem maximizes $v_{\mathrm{T}} x_{d}$ for a given $T$; denote the value $\max v_{\mathrm{T}} x_{d}$ by $v_{\mathrm{T}} x_{d}$. The QF problem minimizes $T$ with its flow value $v_{\mathrm{T}} x_{d} \geq B$ for a given $B$; denote the minimum time $\min T$ for sending the given value $v_{\mathrm{T}} x_{d}$ by $T\left(v_{\mathrm{T}} x_{d}\right)$. The EAF problem maximizes the value $v_{\theta} x_{d}$ for all $\theta \epsilon \mathbf{T}$. An optimal solution to this general EAF problem also solves the MDF and QF problems. For given time $T$ and an ordered set of the terminals, the LMDF problem is to find a feasible flow that lexicographically maximizes the amount leaving each terminal in the given priority order. The MDF, LMDF, QF, EAF and minimum cost dynamic evacuation problems are denoted by MEP, LMEP, QEP, EAEP and MCEP, respectively.

Suppose that $x_{s}$ be a static flow and it has a standard decomposition into a set of chains $\Gamma=\left\{\gamma_{1}, \ldots, \gamma_{r}\right\}$ with $r \leq m$ that satisfies $x_{s}=\sum_{k=1}^{r} \gamma_{k}$, where all chains in $\Gamma$ start and end at the terminal nodes and use the arcs in the same direction as $x_{s}$ does. The lengths of all 
chains $\gamma_{k}$ satisfy $\tau\left(\gamma_{k}\right) \leq T$ for standard chain decomposition $\Gamma$. Then, $\Gamma$ computes a feasible dynamic flow obtained by summing the dynamic flows induced by each chain flows, (Ford and Fulkerson, 1962).

\subsection{Continuous model}

We consider the continuous time interval $\mathbf{T}=[0, T]$, for the given time $T$. Let $x_{c}: A \times \mathbf{T} \rightarrow \mathrm{R}^{+}$be the rate of flow on an arc $e$, where $x_{c}(e, \theta)$ denotes the amount of flow that enters arc $e$ at continuous time $\theta$. The flow rate capacity constraint and the flow conservation constraints are represented by (5-7), similar to the discrete dynamic flow, with the sum over time replaced by an integral.

$$
\begin{array}{ll}
\int_{0}^{\theta} \sum_{e \in B(v)} x_{c}(e, \sigma-\tau(e)) d \sigma-\int_{0}^{\theta} \sum_{e \in A(v)} x_{c}(e, \sigma) d \sigma \geq 0, & \forall v \notin\{s, t\}, \theta \in \boldsymbol{T} \\
\int_{0}^{T} \sum_{\substack{T \\
0 \leq B(v)}} x_{c}(e, \sigma-\tau(e)) d \sigma-\int_{0}^{T} \sum_{\substack{e \in A(v) \\
0 x_{c}(e, \theta) \leq b_{A}(e, \theta),}} x_{c}(e, \sigma) d \sigma=0, \quad \forall v \notin\{s, t\} \\
\forall e \in A, \theta \in \boldsymbol{T}
\end{array}
$$

The objective function is represented by (8) as follows.

$v_{\theta} x_{c}=\int_{0}^{\theta} \sum_{e \in A(s)} x_{c}(e, \sigma) d \sigma=\int_{0}^{\theta} \sum_{e \in B(t)} x_{c}(e, \sigma-\tau(e)) d \sigma$

The different variants of continuous maximum and quickest evacuation problems are defined analogously to their discrete versions. Different variants of approaches with extended characteristics on the parameters and functions are summarized in Section 3.2.

\subsection{Natural transformation}

Fleischer and Tardos (1998) presented a natural transformation for many discrete dynamic flows based on chain decomposable flows to transform into continuous dynamic flows and proved its optimality.

Let $x_{s}(e): \mathbf{T} \rightarrow \mathrm{R}^{+}$and $x_{c}(e): \mathbf{T} \rightarrow \mathrm{R}^{+}$be the amount and the rate of flow on an arc $e$, respectively. Thus, $x_{s}(e, \theta)$ and is the amount of flow on $\operatorname{arc} e$ at discrete time $\theta$ and $x_{c}(e, \theta)$ is the amount of flow that enters arc $e$ at continuous time $\theta$. The former gives a dynamic flow $x_{d}$ on the arc $e$ at discrete time $\theta$ (Kotnyek, 2004) relates these two functions in continuous and discrete models as follows.

$x_{d}(e, \theta)=\int_{\sigma=0}^{\tau(e)} x_{s}(\theta-\sigma) d \sigma$, and $x_{d}(e, \theta)=\sum_{\sigma=0}^{\tau(e)-1} x_{s}(\theta-\sigma)$

The difference between the discrete time approach and the continuous time approach depends upon the flows on arcs. The flow entering an $\operatorname{arc} e$ at time $\theta-\tau(e)$ has already 
arrived at the head node by $\theta$ in discrete approach, however it is still on the arc at that moment in continuous approach.

For $x_{d}(e, \theta)$, a feasible discrete flow entering arc $e \in A$ at time $\theta$ for $\theta=0,1, \ldots, T-$ 1 , we set the continuous flow rate $x_{c}(e, \sigma)$, to $x_{d}(e, \theta)$, for $\sigma \epsilon[\theta, \theta+1)$. From this natural transformation, we obtain a feasible continuous flow and the flow value in any integral interval $[\theta, \theta+i), \theta=0,1, \ldots, T-1, i \in N$ will be same for both $x_{d}$ and $x_{c}$.

\section{Optimization Algorithms}

The dynamic flows have been modelled with finite or infinite time horizons, where the flow must be completed before a given time bound $T$. Time can be discretized such as $\theta=0,1, \ldots, T$ if one is converting a continuous to a discrete model. The parameters on arcs or at nodes can be time or flow dependent rather than fixed. An integrated model can be found in (Koch et al., 2011).

\subsection{Discrete flows over time}

A temporally repeated solution for the single-source single-sink MDF problem is obtained in polynomial time in the network $\mathcal{N}=\left(V, A, b_{A}, \tau,\{s, t\}, T\right)$, (Ford and Fulkerson, 1958). The discrete dynamic flows in the network $\mathcal{N}$ are equivalent to the static flows in the time-expanded network $\mathcal{N}_{T}=\left(V_{T}, A_{M} \cup A_{H}\right)$. The pseudopolynomial algorithms based on $\mathcal{N}_{T}$ are polynomial time in $T$ therefore, only pseudo-polynomial in $\mathcal{N}$. The multi-terminal problems are either solvable in $\mathcal{N}_{T}$ or with flows allowed in opposite directions (Hoppe and Tardos, 2000). However, the multi-terminal problems with fixed demand-supply vectors at terminal nodes have polynomial time decomposition.

The LMDF solution finds a feasible flow for a given time $T$ that lexicographically maximizes the flows leaving the given ordered terminals $s_{1}, \ldots, s_{k}$. For LMDF, Minieka (1973) and Megiddo (1974) give time-expanded network based algorithms and Hoppe and Tardos (2000) polynomially solve the general problem in original graph instead of timeexpanded graph. Using the bisection or the parametric search via the related MDF problem, Burkard et al. (1993) solve the quickest $s-t$ flow problem without holdover flows. A nonstandard chain decomposition solves the general QF in polynomial time $\log T$, (Hoppe and Tardos, 2000). Authors (Miller-Hooks and Patterson, 2004; Koehler et al. 2002) adopt the time-varying inputs in the generalized time-expanded network for the QF. The EAF $s-t$ problem has been solved pseudopolynomially by calculating the successive shortest paths for min-cost flow, (Wilkinson, 1971; Minieka, 1973). The EAF problem for multi-sources and a single-sink is solved with strongly polynomial time algorithm in the input plus output size, (Baumann and Skutella, 2009).

The contraflow evacuation (CFE) problem is to find a network reconfiguration with ideal directions to each lane so that a given objective function is optimized subject to the given constraints. Strongly polynomial time algorithms for the problems of maximizing a static flow and a dynamic $s-t$ flow are presented in (Rebennack et al., 2010). But, this problem is $N P$-hard in general. The general quickest CFEP is $N P$-hard. The earliest arrival CFEP is polynomial time solvable for two-terminal series parallel graphs, two terminal 
general graph, multi source and/or sink networks and on lossy networks, (Dhamala and Pyakurel, 2013; Pyakurel et al. 2014; Pyakurel and Dhamala, 2014a; 2015; 2015a).

\subsection{Continuous flows over time}

Assuming that the cost, upper bounds and rates of demand or supply in each arc are piecewise analytic, there exists a piecewise analytic solution, provided the feasible region is nonempty and bounded, (Anderson et al. 1982). They consider the continuous MDF with zero travel times and time dependent capacities and extend the max-flow min-cut theorem to cuts in the continuous case. By computing a MDF for each source to the sink, Hajek and Ogier, (1984) solve a special case of continuous QF problem with multi-sources, a single-sink, and zero travel times on the arcs. A result in (Philpott, 1990) extends the results of (Anderson et al. 1982) to arbitrary travel times and presents triple optimization results of MDF, QF and minimizing the total egress time, similar to discrete approach given in (Jarvis and Ratliff, 1982). The generalized cut capacity bounds the value of the continuous flow, (Philpott, 1990). The weak and strong duality relations hold, (Pullan, 1993). Fleischer and Tardos (1998) establish several polynomial time algorithms by natural transformations of discrete flows to continuous ones.

Fleischer, L. (2001a) considers the universally maximum flow with at most $k$ piecewise constant arc and node capacities to change over time and improves the previous algorithm by a factor of $O(n k)$. A continuous linear program for general continuous networks with cost minimization has been formulated given bounded measurable functions of cost, upper bounds, rates of demand or supply and levels of storage in each node, see (Hamacher and Tjandra, 2002).

For the integral time horizon $T$, the natural transformation of chain decomposable flows are feasible. If $T$ is not integral, then we have a discrete $\lceil T\rceil$ time. Due to the integral transit time, all the chain flows have integral length. This results that the chain flows used with time horizon $\lceil T\rceil$ can also be used with time bound $T$. A chain flow is not affected by the time it starts to use an arc. But it reduces the time that the chain flow uses the arc. Thus, a discrete $\lceil T\rceil$-horizon chain decomposable flow can be transformed naturally into a continuous $T$-horizon flow and stop sending flow along each chain flow $\gamma$ at time $T-\tau(\gamma)$ instead of time $\lceil T\rceil-\tau(\gamma)$. In dynamic flow a chain induces the same amount of flow at each chain flow. Hence if the discrete chain decomposable flow is feasible then the continuous chain decomposable flow is also feasible, see (Fleischer and Tardos, 1998).

\subsection{Minimum cost flow problems}

The minimum cost evacuation problem (MCEP) with arc costs relates to the minimum cost flow problem sending a fixed amount of flow in a given time with minimum total cost. Both the minimum cost MDF and the minimum cost QF are NP- hard, (Klinz and Woeginger, 1995). They show that finding a minimum cost temporally repeated flow is strongly $N P$ - hard. The minimum cost $s-t$ flow over time is pseudo-polynomially solvable, (Baumann and Skutella, 2009). Fleischer and Skutella, (2003) consider the problem of minimizing the cost over time without intermediate storage in time-expanded networks. They prove that finding a minimum cost flow does not require intermediate storage, provide an approximation scheme, and a capacity scaling FPTAS when the costs are proportional to the transit times. 
Cai et al. (2001) propose an algorithm for the single-source single-sink minimum cost flow problem with time-varying travel times, capacities and arc costs. An infinite time horizon minimum cost flow problem over time with an objective of maximizing throughput has been polynomially solved in (Orlin, 1984). But this approach cannot address the important changing issues over time; especially, the number of evacuees entered into the system and the starting and stopping points of the process. Pardalos and Arulselvan (2009) show that the fixed switching cost (costs on the arcs resulting from switching an arc in an arc reversal process) CFE problem is equivalent to the 0-1 minimum improvement flow problem.

\section{Contraflow Configuration}

\subsection{Heuristic methods}

The methods which rely on user-provided evacuation time upper bounds do not perform well for large scale networks. Many heuristics are designed for approximate minimum egress times compromising to minimum computational costs. Most of the earlier heuristics do not capture the essence of route capacity constraints, which greatly influence evacuation plans whenever the number of evacuees dramatically increases and the network gets complex. The single route capacity constrained planner (SRCCP) and multiple route capacity constrained planner (MRCCP) are tested in building evacuation networks, (Lu et al., 2003). Lu et al., (2005a) improve the MRCCP for larger networks, the capacity constrained route planner (CCRP), with time complexity $O(p n \log n)$. The improved CCRP in (Lu et al., 2006) has a better time complexity $O\left(p\left(m+2 n \tau_{\max }\right)\right)$, where $\tau_{\text {max }}$ denotes the maximum travel time, in comparison to LP based methods and the earlier CCRP itself. With an objective of minimizing the computational cost of obtaining a very large evacuation route plan, Kim et al. (2007) improve the CCRP by intelligent load reduction (ILR) and incremental data structure (IDS).

George et al. (2007) and George and Shekhar (2006) study spatio-temporal networks, whose topology and parameters change over time, modeled as a time aggregated graph. This graph with time series data reduces the complexity and uses less memory in comparison to the time expanded graphs. The former propose two algorithms to compute shortest paths for fixed start time and best start time. Zhou et al. (2010) study the CCRP for the earliest arrival time with a spatial network database advancing the idea of time aggregated graph. The CCRP gives the number of evacuees followed by the calculation of the earliest arrival source-sink travel time for a given start time. This performs better than the optimal time expanded graph approach like being used by other CCRP.

A macroscopic model addressing road capacity constraints, multiple sources, congestion and scalability is given in (Kim et al., 2008) for the contraflow minimizing the egress time. Their greedy and bottleneck relief heuristics improve the evacuation egress time by at least 40 percent. The hardness is a function of the overload degree, i.e., the ratio of the number of travelling units to the bottleneck capacity without contraflow. They show that the problem of minimizing the evacuation egress time in the general network is $N P$-hard.

Many affords have been made in dealing with integrated contraflow approach (cf. Section 4.2). 


\subsection{Integrated Approaches}

To deal the problem of traffic jam at intersection, a crossing elimination strategy has been developed in (Cova and Johnson, 2003). The lane based contraflow and crossing elimination strategies introduced in (Xie and Turnquist, 2011) support each other by increasing capacity in specific directions. These combined action of discrete network capacity and connectivity setting results a combinatorial complexity. To overcome this, an integrated Lagrangian relaxation and tabu search solution method has been developed. The former decomposes the problem into simpler pieces, and the latter solves the most complex of these pieces. They tested the algorithm using an evacuation network located in Monticello, Minnesota with fixed number of terminals and full lane reversal of transportation network.

To deal with contraflow at road segments and crossing elimination at intersections jointly, a bi-level, lane-based network optimization and simulation model has been formulated in (Xie et al., 2010). The upper level optimizes the network evacuation performance subject to the contraflow and crossing-elimination constraints and the lower level simulates dynamic evacuation flows. The computational complexity is combinatorial. They developed a Lagrangian-relaxed and tabu-based search solution methods which reduces the structural complexity and avoids the local optimality, respectively. A large scale case study has been presented taking the Monticello nuclear plant at the Monticello, Minnesota. The contraflow problem including more realistic situations such as important evacuation priorities and the setup time for the contraflow operation have been considered in (Wang et al., 2013). Their two-layer optimization model that considers the setup time uses a modified particle swarm optimization based algorithm that solves the problem efficiently on singlesource and multi-sinks network.

A contraflow network reconfiguration giving opportunity of root choice for evacuees has been presented in (Lv et al., 2010). They ignored the background traffic and did complete contraflow reconfiguration that does not allow the emergency vehicle back into the evacuation area. They address the character of root manager and evacuees into a bi-level programming, where the upper level model minimizes the total evacuation time of all evacuees and the lower level bases on user equilibrium assignment. They used the solution method based on discrete particle swarm optimization and Frank-Wolfe algorithm improving evacuation efficiency and decreasing evacuation time from 30 to 60 percent.

The contraflow routing problem based on the maximum throughput flows and on reversed shortest paths has been introduced in (Min et al., 2013). The throughput of flows on an evacuation path is the total amount of flow over the evacuation time. In this problem, the most important factor is the path finding time in order to reduce the computation time. By running only one shortest path-equivalent algorithm, all the shortest paths from all source nodes to destination nodes have been found which reduce the path finding time significantly. They presented an efficient and highly scalable algorithm based on maximum throughput flows utilizing the reverse shortest path algorithm to reduce the path finding time.

A multi-modal evacuation planning problem on single-source and multi-sinks network including the simultaneous consideration of contraflow, repair of roads and 
classification of evacuees according to their needs has been introduced in (Wang et al., 2010). Three layer methods have been used for the solution of integrated design and planning problem. Experimental result shows that on the damaged transportation network, the evacuation time has been reduced with more than $50 \%$ by constructing one new road and with $20 \%$ by re-planning the resource.

Hua et al. (2014) study a multi-modal integrated contraflow model for uncertain arrivals of evacuees in an evacuation region with low mobility population that has little access to personal vehicles. The integrated contraflow strategy, whose solution procedure has been developed in two-levels, is based on the fact that the transit-based evacuees and the auto-based evacuees will be evacuated to different sinks. Firstly, solving the mixed-integer linear programming formulation similar to the minimum-cost flow problem, the transit based people are evacuated by using branch and bound algorithm. Secondly, the bi-level model of auto-based evacuation problem has been solved approximately using the genetic algorithm. They presented a valid and useful case study for a super typhoon on an evacuation network. The computational results on the case study has been justified the validness and usefulness of their integrated contraflow model for evacuation planning problem.

\subsection{Analytical methods}

Rebennack et al. (2010) analytically study network flow problems with arc reversal capability. They solve the two-terminal maximum dynamic contraflow (MDCF) problem with discrete time setting. The MDCF problem asks for the maximized flow value through it if, in addition, the reversals of arc directions are allowed at time zero. Let the reversal of an arc $e=(v, w)$ be $e^{-1}=(w, v)$. Given a dynamic network $\mathcal{N}$ with symmetric travel times, the auxiliary dynamic network $\overline{\mathcal{N}}=\left(V, E, b_{E}, b_{V}, \tau, S, D, T\right)$ consists of the modified arc capacities and travel times, respectively, as

$$
b_{E}(\bar{e})=b_{A}(e)+b_{A}\left(e^{-1}\right), \quad \text { and } \tau(\bar{e})=\left\{\begin{array}{cc}
\tau(e) & \text { if } e \in A \\
\tau\left(e^{-1}\right) & \text { otherwise }
\end{array}\right.
$$

where, an edge $\bar{e} \in E$ in $\overline{\mathcal{N}}$ if $e \mathrm{~V} e^{-1} \in A$ in $\mathcal{N}$. The remaining graph structure and data are unaltered.

Their algorithm (cf. Algorithm 1) determines a dynamic temporally repeated flow on the auxiliary graph with modified capacity and travel times.

Algorithm 1. (Rebennack et al., 2010) Maximum dynamic contraflow algorithm

1. Given a two terminal network $\mathcal{N}=\left(V, A, b_{A}, \tau, s, t, T\right)$ with integer parameters.

2. Obtain the auxiliary network $\overline{\mathcal{N}}=\left(V, E, b_{E}, \tau, s, t, T\right)$.

3. On $\overline{\mathcal{N}}$ run a temporally repeated flow algorithm.

4. Decompose the obtained flow from Step 3 into path and removable cycles

5. An arc $(w, v) \in A$ is reversed if and only if the flow along arc $(v, w)$ is greater than $b_{A}(v, w)$, or if there is a nonnegative flow along arc $(v, w) \notin A$ and the resulting flow is EAF with the arc reversals for the graph $\mathcal{N}$.

6. Obtain a MDF with the arc reversals for the network $\mathcal{N}$. 
Theorem 1. (Rebennack et al., 2010) The $s-d M D C F$ problem can be solved in time $O\left(h_{2}(n, m)+\right.$ $\left.h_{3}(n, m)\right)$, where $h_{2}(n, m)=O(n . m)$ and $h_{3}(n, m)=O\left(n^{2} \cdot m^{3} \cdot \log n\right)$ are the time required for the flow decomposition and the MSF problem, respectively.

With natural transformation, we solve the maximum continuous dynamic flow (MCDCF) problem on two terminal network $\mathcal{N}=\left(V, A, b_{A}, \tau, s, t, T\right)$, see (Pyakurel and Dhamala, 2015b). The problem seeks to find a maximum flow that can be sent from the source to the sink within time interval $[0, T]$. We replace the Step 3 of Algorithm 1 with minimum cost flow algorithm of (Fleischer and Tardos, 1998) with flow rate $x_{c}(\bar{e})$, capacity $b_{E}(\bar{e})$, and transit time $\tau(\bar{e})$. The other steps remain the same. The obtained flow from our algorithm is the MCDF with arc reversal capability for the network $\mathcal{N}$. The solution can be obtained with the same complexity as in the MDCF problem of (Rebennack et al., 2010)

Problem 1. Given $\mathcal{N}=(V, A)$ having $\tau(e) \in \mathbb{Z}^{+}$with $\tau(e)=\tau\left(e^{-1}\right)$ and capacity $b_{A} \in \mathbb{Z}^{+}$on each $e \in A$, the earliest arrival contraflow (EACF) problem requires the maximum amount of flow that can be sent in every time period $0 \leq \theta \leq T$ for discrete time model, and in each time point $\theta \in[0, T]$ for continuous time model, from the sources to the sinks with arc reversal capability at time zero.

For a particular two terminal series parallel (TTSP) network, the EACF problem is solved in (Dhamala and Pyakurel, 2013). By constructing the auxiliary network $\overline{\mathcal{N}}=(V, E)$ of TTSP network $\mathcal{N}=(V, A)$ and replacing the Step 3 of Algorithm 1 with minimum cost circulation flow algorithm of (Ruzika et al., 2011), Algorithm 2 is obtained, see also (Pyakurel and Dhamala, 2014a).

Algorithm 2. (Dhamala and Pyakurel, 2013) Earliest arrival contraflow on TTSP networks

1. On TTSP auxiliary network $\overline{\mathcal{N}}=\left(V, E, b_{E}, \tau, s, t, T\right)$, replace Step 3 of Algorithm 1 by algorithm of (Ruzika et al., 2011) to compute the minimum cost circulation flow.

2. Obtain the EACF solution on TTSP networks.

Theorem 2. (Dhamala and Pyakurel, 2013) An earliest arrival contraflow problem on TTSP network can be solved in $O(n m+m \log m)$ time complexity.

With natural transformation of (Fleischer and Tardos, 1998), we solve the continuous earliest arrival contraflow problem on TTSP networks in continuous time setting. This problem can be solved in the same complexity as in the EACF problem on TTSP networks. For continuous contraflow model we refer to (Pyakurel and Dhamala, 2015b).

Moreover, the EACF problem can be solved in two-terminal general network (Pyakurel and Dhamala, 2015). However, it takes pseudo-polynomial time iterations. In order to evacuate the evacuees in given priority orderings, Pyakurel and Dhamala (2015) introduce lexicographically maximum dynamic contraflow problem and solve it with polynomial time complexity. For the foundation of evacuation planning, static contraflow problems are also presented, see (e.g. (Rebennack et al., 2010; Pyakurel and Dhamala, 2015). Moreover, the earliest arrival transhipment contraflow problem in multi source and/or multi sink networks has been solved in (Pyakurel and Dhamala, 2015a).

In the generalized evacuation network where the maximum evacuees are evacuated with minimum lost of life and property, generalized contraflow approach has been developed in (Pyakurel et al., 2014). They solve the generalized maximum dynamic contraflow problem as well as the generalized earliest arrival contraflow problem on two-terminal lossy network. 


\section{Facility Location-Allocation}

Hamacher et al. (2011) and Heller and Hamacher (2011) combine evacuation problems with location analysis to predict and evaluate evacuation plans. Hamacher et al. (2011) introduce the facility location model approach to reduce the evacuation time by minor changes. However, good decision of locating emergency units or commercial facilities in proper places remains a challenging issue. Consider the discrete time MEP given by a graph $\mathcal{N}=\left(V, A, b_{A}, \tau, s \cup t\right)$. Let $L \subseteq A$ be the set of feasible locations, $F$ be the set of all facilities, $r: F \rightarrow N$ be the size of facilities and $q: L \rightarrow N$ be the number of facilities that can be placed on the possible locations. Define $b_{A}^{l}(e)=b_{A}(e)-\max _{f \in F}\{r(f) \mid \operatorname{loc}(f)=e\}$ where loc: $F \rightarrow L$ denotes an allocation of the facilities to the edges. The flow location problem (FlowLoc) asks to determine an allocation loc such that the $s-t$ flow value in the network $\mathcal{N}_{\text {loc }}=\left(V, A, b_{A}^{l}, \tau, s \cup t\right)$ is maximized. It is important to maintain as many sourcesink paths as possible while placing a facility on the location edges for the better utilization of the evacuation network. The single facility 1 -FlowLoc problem asks for the location $l=$ $\operatorname{loc}(f) \epsilon L$ of a single facility $f \epsilon F$ with size $r(f)$ such that the reduction of the maximum flow value in $\mathcal{N}=\left(V, A, b_{A},\{s \cup t\}\right)$ is minimal. An allocation of a facility of a given size reduces the capacity of the arc by the respective size.

Given a directed network with locations $L$ and a facility $f$ with size $r(f)$, Hamacher et al. (2011) present three exact algorithms to solve the 1-FlowLoc $s-t$ problem. Their first algorithm compares all maximal flows resulting from all possible facility allocations. As for each possible location one maximum flow has to be calculated, this iterative algorithm takes $O\left(|L| n^{3}\right)$ time by applying one of the variants of push-relabel algorithms of complexity $O\left(n^{3}\right)$ for the flow problem. If the residual capacity is large enough to host the facility $f$, it suffices to perform a single maximum flow calculation in the original network, plus residual capacity checking of all locations, to find an optimal solution to 1-FlowLoc $s-t$ problem; this yields an $O\left(n^{3}+|L|\right)$ algorithm, reducing the computational complexity in practice. However, it takes the same time complexity as that of the iterative algorithm in the worst case when the residual capacity is not large enough. They also improve it by a second modification with redirected flow if the preprocessing is unsuccessful. In this modification, the flow on the edges hosting a location is redirected and checked if enough capacity is generated to host the facility. If it is not the case, the facility is located on the edge which gives the smallest change on the maximum flow value. It takes $O\left(|L| \mathrm{nm}^{2}\right)$ time for this advanced redirect algorithm. Numerical results show that both modified algorithms work better than the iterative one in general, with much smaller run times.

The multi-facility $q$-FlowLoc problem asks for the locations of $q$ facilities $f \epsilon L$ with size $r(f)$ such that the reduction of the maximum flow value in $\mathcal{N}=\left(V, A, b_{A}, \tau, s \cup t\right)$ is as small as possible, where at most $q(e)$ facilities can be allocated on an arc $e \epsilon L$. Obviously, the extensions of the iterative algorithm for solving 1-FlowLoc static $s-t$ problems to $q$ FlowLoc static $s-t$ problems are not polynomial time extendable with $q$ being part of the input. The decision version of the $q$-FlowLoc problem in $\mathcal{N}=\left(V, A, b_{A},\{s \cup t\}\right)$ is polynomial reducible to $3-S A T$, and the considered problem turns to be $N P$-hard (Hamacher et al. 2011). They show that a finite constant $\alpha$-approximation algorithm is impossible for its solution, unless $P=N P$. The problem has also been formulated as a mixed integer program. A computationally faster heuristic for the reduction of flow values and the 
placement of facilities has been proposed, and numerically tested using CPLEX. With a precalculated maximum flow $x_{s}$ in the original network, the edge $e \epsilon L$ having the largest residual capacity $u(e)-x_{s}(e)$ has been facilitated with the next $q(e)$ facilities by decreasing order of facility sizes. The maximum flow is reduced by a recalculated maximum flow on $e$ if $u(e)-x_{s}(e) \geq r\left(f_{i+j-1}\right)$, for $j=1, \ldots, q(e), i=1, \ldots, q$, until all facilities are placed.

The dynamic $s-t$ 1-FlowLoc problem can be solved by either allocating the facilities on all time-copies arcs in the time-expanded network, or making use of the minimum cost circulation solution in the temporally repeated flows. The iterative algorithm and its modification with respect to residual capacity preprocessing for the static $s-t 1$ FlowLoc problem is still valid for the dynamic $s-t 1$-FlowLoc problem. In (Hamacher et al. 2011), an integer programming formulation is given for the dynamic $s-t q$-FlowLoc problem. Like in the static case, an $\alpha$-approximation algorithm is impossible for its solution unless $P=N P$. The static version of the above heuristic can be applied to the corresponding dynamic problem by calculating a maximum flow via minimum cost calculations. An alternative heuristic for the dynamic $s-t q$-FlowLoc problem makes use of the dynamic $s-t$ 1-FlowLoc solution such that $q(e)$ facilities with the largest size have to be placed on the arc $e$ which takes the facility in the optimal 1-FlowLoc solution. The process iterates until all facilities are placed (Hamacher et al. 2011). Heller and Hamacher (2011) give an integer programming formulation of the $N P$-hard multi-terminal $q$-FlowLoc problem and presents a heuristic for all feasible solutions that could be obtained by calculating a maximum flow in a special $s-t$ network representing a transportation problem. If the flow obtained is also a minimum cost flow, different versions of the heuristic have been proposed using different cost functions on the edges of the special network, depending on the graph and the problem type to gain the feasible solutions faster.

\section{Simulation Practices}

Agent based micro-, macro-, and meso-simulators have been used to visualize the evacuation scenarios over time, study the current plans, and identify inconsistencies of the analytical models for large-sized evacuation networks. These traffic simulations seek to overcome practical difficult for urgent realization of the optimization models, although various advantages and disadvantages exist among them, (Pid et al., 1993). For instance, micro-simulators taking care of individual vehicles are suitable to predict and adopt to the changes in the model, but experience heavy computational cost when the system gets larger and the vehicles enter in large lots. PARAMICS, CORSIM, VISSIM, EGRESS, and FlightSim are the most widely adopted microsimulation software tools for evacuation and general traffic management studies. Most of the micro-model studies seek more accuracy in simulation performance; see for instance, (Pardalos and Arulselvan, 2009; Kotusevski and Hawick, 2009) for the comments and references.

The software CORSIM which combines the two microsimulation models NETSIM, and FRESIM for surface streets and freeways, respectively, $\mathrm{h}$ cas applications in emergencies. The CORSIM which includes features such as altering signals, traffic diversions, access restrictions and roadway clearance has been tested on different traffic events, simulation of available plans and evacuation situation (Sisiopiku et al. 2004). The models based on CORSIM which also includes speed, queuing time and length do not give 
the best test results in real-world problems, though. This model has been used to test the effectiveness of contraflow to establish better evacuation routes. There exists research based on this simulation approach reporting computationally efficient in obtaining quick results, e.g., (Williams et al. 2007).

The micro-traffic simulation software VISSIM is also popular in evacuation network scenarios. In addition, these models have been implemented to compare the results with lane reversals and without them, with efficiency measures as throughput at key nodes, queue lengths and average speeds. As a result the lane reversals perform better with increasing throughput and decreasing queue lengths, e.g. (Tagliaferri, 2005).

PARAMICS has been used to simulate high-risk evacuation areas and to compare the effectiveness of simultaneous and staged evacuation strategies, e.g. (Chen and Zhan, 2008). Although it has the benefit of different alterations like numbers of vehicles, traffic control, alternate exits and critical links, the assumptions that require a priori known route choices and destination information limit the model in real-evacuation contexts. Because of the drivers' interest to use shortest paths, resulting in frequent congestion, Cova and Johnson (2002) suggest a staged evacuation strategy based on high population density affected zones.

The software tools EGRESS and FlightSim for evacuation in emergencies make use of cellular automata as a method of discrete microsimulation. Cellular automata consider a regular finite-dimensional lattice partitioned into discrete cells with a discrete step time evolution. This approach divides the evacuation region into accessible and non-accessible cells of fixed and equal size; each is assigned a value from a discrete finite set. An update will be made based on the values of its neighbourhood at the preceding time step with respect to deterministic or probabilistic local rules defined to control the movements of evacuees. The methods take advantage of more freedom of the individual evacuees on the routes contrary to vehicle movements. These simulators exploiting the cellular automata design partially take some behavioural effects of the evacuees into account, e.g., (Kluepfel et al. 2000; Doheny and Fraser, 1996; Hamacher and Tjandra, 2002). Kneidl et al. (2010) couple a microscopic model based on cellular automata with a macroscopic model based on dynamic network optimization. The output of each model is fed into the other for different configuration setups to narrow down the gaps on evacuation times approximated by them. The test has been limited to a small corridor and is not deeply analyzed with respect to its theoretical aspects.

Davidich and Koester (2012) gain more realistic and reliable predictions by proposing fast methods which automatically calibrate the simulation of pedestrian streams. They also present references for evacuation simulations which make use of different automata to reduce the disadvantages of cell discretization. Pel et al. (2012) present a survey on traffic simulation models with mathematical formulations that have been used in evacuation planning in emergencies. They discuss, with lists of evacuation software, the effect of evacuees' behaviour in emergency planning and realization. The early evacuation traffic simulation models have been analyzed with their characteristics and applications.

Kimms and Maassen (2011) consider the evacuation problem in urban areas and test a created example with the objective to minimize the total number of vehicles in more 
dangerous areas. They interlink optimization and simulation methods by making use of an optimization based simulation procedure in which the optimization model for computing possible traffic routes and assignment of safe destinations in complex evacuation systems already makes use of the cell transmission model of (Daganzo, 1995). Following the optimization model, the software DOSIMIS-3 Simulator predicts the traffic evolution (Kimms and Maassen, 2011).

The macro-models which are better applicable in large scale evacuation networks with respect to computation time and space have rather been used to simplify the application and analysis, than to improve the accuracy. These models study the aggregated traffic and aggregated flow through model variables such as flow rate, speed and density, earlier models include MASSVAC of (Radwan et al. 1985) and NETVAC of (Sheffi et al. 1980) which have been popular since the 1980's. The former approach can be applied to simply-structured rural networks, whereas the latter can be applied to radial evacuation from risk zones, as may be the case in a nuclear accident. The macrosimulators OREMS, DYNEV and ETIS additionally incorporate more advanced features making the models more appropriate, and suggest the evacuation response factors to improve them, (Moriarty et al. 2007)

The mesosimulators, for example, CEMPS, SmartCAP, SmartAHS, DYNEMO have been introduced as another generation of software with view of keeping advantages over disadvantages of the macro-micro simulators. However, the macro-micro software tools have not been replaced instead; see (Pardalos and Arulselvan, 2009) for reviews.

\section{An Application}

Differentiating the vehicle routine problem, Bish (2011) gives an integer programming model for bus-based evacuation problem and presents an efficient heuristics to the modified model for the bus assignment with given routes of evacuation. Goerigk and Gruen (2014) present a tabu search and compare the experiments with CPLEX results for the robust bus evacuation problem. They present two phases of integer programming with given scenarios. With known number of evacuees at the beginning of the evacuation process, Goerigk et al. (2013) propose several algorithms for upper bounds and lower bounds for the optimum and compare the CPLEX outputs to the branch and bound algorithm they they presented. All of these approaches consider the minimization of evacuation time and highlight the importance of transit-dependent evacuees needs.

Goerigk et al. (2013) and Goerigk and Gruen (2014) apply the branch and bound algorithms and a tabu search heuristic for transit-based evacuation scenario. The branch and bound algorithms solve the bus-based transportation problem, with given number of evacuees at the sources, for the minimal duration of evacuation by routing and scheduling. The tabu search heuristic solves the robust bus-based evacuation planning problem, where the number of evacuees is not known exactly but a set of estimates for the number of evacuees at each source is given.

These algorithms are used for a case study in Kathmandu - the metropolitan capital city of Nepal (cf. Figure 2), (Pyakurel, et al. 2014a; 2015) where the population around 25,672 within the area $1.45 \mathrm{~km}^{2}$ has been evacuated. They have used 100 or 140 buses of 
uniform capacity that may carry 60 or 90 people at a time with comfortable or adjustable travel. The road speed has been assumed to be $12 \mathrm{~km} / \mathrm{hr}$ or $15 \mathrm{~km} / \mathrm{hr}$ for the buses. Among all, only $50 \%$ or $60 \%$ population have been evacuated to 4 or 5 safe destinations from 5 or 6 pickup locations. The algorithms branch and bound and tabu search have been tested by executing 2.2.2.2.2.2 = 64 instances for 15 minutes each.

The branch and bound algorithms yield the best evacuation time as 29 minutes (respectively, 36 minutes) at 6 or 5 sources and 5 sinks for 50 percent population (respectively, 60 percent population) using 140 buses having 90 evacuees per bus capacity and $15 \mathrm{~km} / \mathrm{hr}$ speed (cf. Figure 3). For the tabu search heuristic, the best evacuation time is 44 minutes at 5 sources and 5 (or 4 sinks) for 50 percent (or 60 percent population), using 140 buses having 90 evacuees per bus capacity and $15 \mathrm{~km} / \mathrm{hr}$ speed (cf. Figure 4).

We can use these results to derive suggestions for high-level planning strategies for practitioners in the Kathmandu area. The branch and bound results can be implemented as emergency response as these can be tested in much faster time, however, the tabu-search results can be considered as planning issues.

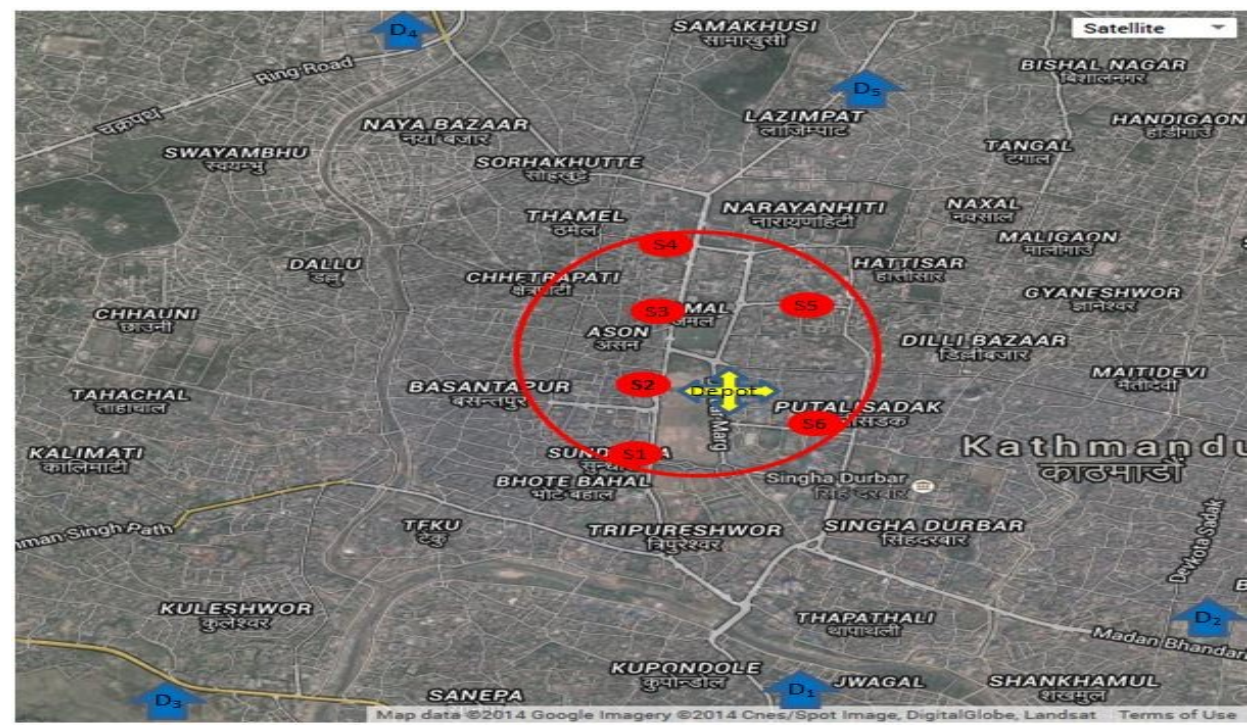

Figure 2: Map of core city Kathmandu

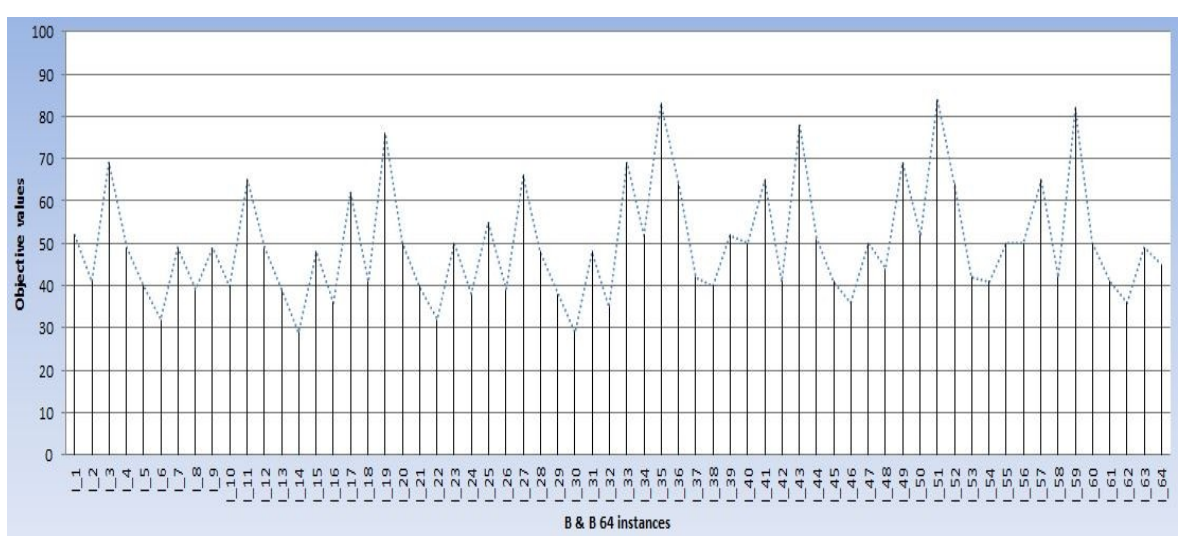

Figure 3: Case study overview for BBEP using branch and bound 


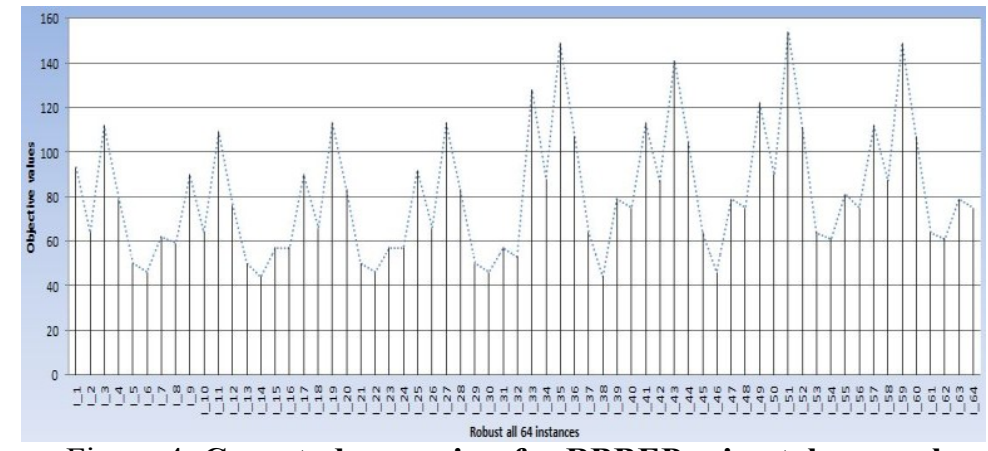

Figure 4: Case study overview for RBBEP using tabu search

\section{Conclusions and Further Works}

We analyzed most of the mathematical and simulation approaches of evacuation planning. The relatively young vehicle routine related transit-dependent models play equally important roles as the usual network flow related car-evacuation models. The macro and micro models bound the solutions, but the latter gives more accuracy with time inefficiency. The simulation-optimization hybrid models improve the solution quality by exploiting the advantages of one to the other. Only few cases like single (source, sink) evacuations are polynomially solvable. If supply demand vectors are given in advance then optimum solutions exist with generalized chain decomposition of flows back and forth. Because of the subroutines depending on submodular function minimization, efficient implementations of them are quite unrealistic.

The models that yield exact optimal solutions can be implemented only for smallscale evacuation problems like building evacuation or an event management. Fortunately, a number of approximate methods are presented in the literature applicable for large-scale evacuations. No universal consensus has been drawn on the advantages and disadvantages over existing models. We summarized results with time dependent attributes and continuous flows which yield better results although rather costly. Recently, the evacuation planning problems combined with location analysis are studied to predict and evaluate evacuation plans that have potential in emergency logistics supports. The importance of contraflow in an existing transportation network has been increased over the past years. It improves the solution quite a lot but is hardly studied from analytical point of views. Easy cases of maxflow, QF and earliest arrival CFE problems have been studied.

A number of emerging problem have been explored from this research. We have defined the contraflow problems in case of continuous time framework. However, the evacuation problems that are left unsolved in case of discrete time framework are still unsolved with respect to continuous time frame. On the other hand, if demand-supply vectors at sinks-sources are known in advance, the discrete transhipment problems are solved with certain reasonable time complexity. The corresponding continuous problems are expected to be solved with similar time complexity, even with contraflow. We investigated the contraflow approaches with complete lane reversals; however, contraflow problems with partial lane reversals would be very emerging from the prospective of emergency logistic supports.

We have some limitations in this study that are relevant to highlight. During emergency, we have not considered the flow of emergency vehicles towards disastrous areas. We also assumed that the roads are not damaged after disasters. Moreover, we reverse the 
direction of road segment without any processing cost. Further studies addressing these limitations are of additional importance in evacuation optimization.

Acknowledgements. The first author acknowledges the support of DAAD for his participation at International Conference on Cities, People and Places, Colombo, Sri Lanka, October 26-28, 2015.

\section{References}

Anderson, E.J., Nash, P. and Philpott, A.B. (1982), A class of continuous network flow problems. Mathematics of Operations Research, 7:501-514.

Baumann, N. and Skutella, M. (2009) Earliest arrival flows with multiple sources. Mathematics of Operations Research, 34:499-512.

Bish, D. R. (2011) Planning for a bus-based evacuation. OR Spectrum, 33:629-654.

Burkard, R.E., Dlaska K. and Klinz, B., (1993) The quickest flow problem. ZOR-Methods and Models of Operations Research, 37:31-58.

Cai, X., Sha, D. and Wong, C. K. (2001) Time-varying minimum cost flow problems. European Journal of Operations Research, 131:352-374.

Chen, X. and Zhan, F.B. (2008) Agent-based modelling and simulation of urban evacuation: relative effectiveness of simultaneous and staged evacuation strategies. Journal of the Operational Research Society, 59:25-33.

Cova, T. and Johnson, J. (2002) Microsimulation of neighbourhood evacuation in urban wildland interface, International Journal of Geographical information System, 11:763-784.

Cova, T. and Johnson, J. P. (2003) A network flow model for lane-based evacuation routing Transportation Research Part A: Policy and Practice, 37:579--604.

Daganzo, C.F. (1995) The cell transmission model, part II: network traffic. Transportation Research Part B, 29:79-93.

Davidich, M. and Koester, G. (2012) Towards automatic and robust adjustment of human behavioral parameters in a pedestrian stream model to measured data. Safety Science, 50:1253-1260.

Dhamala, T.N. (2015) A survey on models and algorithms for discrete evacuation planning network problems. Journal of Industrial and Management Optimization, 11:265-289.

Dhamala, T.N. and Pyakurel, U. (2013) Earliest arrival contraflow problem on series-parallel graphs. International Journal of Operations Research, 10:1-13.

Doheny, J.G. and Fraser, J.L. (1996) MOBEDIC - a decision modelling tool for emergency situations. Expert Systems With Applications, 10:17-27.

Fleischer, L. (2001a) Universally maximum flow with piecewise-constant capacities. Networks. 38:115-125.

Fleischer, L. and Skutella, M. (2003) Minimum cost flows over time without intermediate storage. SODA '03 Proceedings of the fourteenth annual ACM-SIAM symposium on Discrete algorithms, Society for Industrial and Applied Mathematics Philadelphia, 66-75.

Fleischer, L. and Tardos, E. (1998) Efficient continuous-time dynamic network flow algorithms. Operations Research Letters, 23:71-80.

Ford, F.R. and Fulkerson D.R. (1958) Constructing maximal dynamic flows from static flows. Operations Research, 6:419-433.

Goerigk, M., Gruen, B. and Hessler, P. (2013) Branch and bound algorithms for the bus evacuation problem. Computers and Operations Research, 40:3010--3020.

Goerigk, M. and Gruen, B. (2014) A robust bus evacuation model with delayed scenario information. $O R$ Spectrum, 36:923-948.

George, B., Kim, S. and Shekhar, S. (2007) Spatio-temporal network databases and routing algorithms: a summary of results. Proceedings of the 11th International Symposium on Spatial and Temporal Databases (SSTD), 460-477.

George, B and Shekhar, S. (2006) Time-aggregated graphs for modeling spatio-temporal networks- An extended abstract. Proceedings of the Workshop at International Conference on Conceptual Modeling.

Hajek, B. and Ogier, R.G. (1984) Optimal dynamic routing in communication networks with continuous traffic. Networks, 14:457-487.

Hamacher, H.W. and Tjandra, S.A (2002) Mathematical modeling of evacuation problems: A state of the art, In: M. Schreckenberger and S.D. Sharma (Eds.), Pedestrain and Evacuation Dynamics, Springer, Berlin, Heidelberg, 227-266. 
Hamacher, H.W., Heller, S. and Rupp, B. (2011) Flow location (FlowLoc) problems: dynamic network flows and location models for evacuation planning. Annals of Operations Research, 207:161-180.

Heller, S and Hamacher, H. W. (2011) The multi-terminal q-flowloc problem: a heuristic. Proceedings of the International Network Optimization Conference, volume 6701 of Lecture Notes in Computer Science, pages 523-528. Springer.

Hoppe, B. and Tardos, E. (2000) The quickest transshipment problem. Mathematics of Operations Research, 25:36-62.

Hua, J., Ren,G., Cheng,Y. and Ran, B. (2014) An integrated contraflow strategy for multimodal evacuation. Hindawi Publishing Corporation Mathematical Problems in Engineering (Volume 2014).

Jarvis, J. and Ratliff, H. (1982) Some equivalent objectives for dynamic network flow problems. Management Science, 28:106-108.

Kimms, A. and Maassen, K.-C. (2011) Optimization and simulation of traffic flows in the case of evacuating urban areas. OR Spectrum, 33:571-593.

Kim, S., Shekhar, S. and Min, M. (2008) Contraflow transportation network reconfiguration for evacuation route planning. Transactions on Knowledge and Data Engineering, 20:1-15.

Kim, S. George, B. and Shekhar, S. (2007) Evacuation route planning: Scalable heuristics. Proceedings of the 15th International Symposium on Advances in Geographic Information Systems ACM GIS, 2007.

Klinz, B. and Woeginger, G.J. (1995) Minimum cost dynamic flows: the series-parallel case. Integer Programming and Combinatorial Optimization, Lecture Notes in Computer Science, Springer Berlin, 920:329-343.

Kluepfel, H., Meyer-Koenig, T., Wahle, J., and Schreckenberg, M. (2000) Microscopic simulation of evacuation processes on passenger ships. Fourth International Conference on Cellular Automata for Research and Industry, Karlsruhe, Germany.

Kneidl, A., Thiemann, M., Borrmann, A., Ruzika, S., Hamacher, H.W., Koester, G. and Eank, E. (2010) Bidirectional coupling of macroscopic and microscopic approaches for pedestrian behavior prediction. Conference Proceeding PED.

Koch, R., Nasrabadi, E. and Skutella, M. (2011) Continuous and discrete flows over time - A general model based on measure theory. Mathematical Methods of Operations Research, 73:301-337.

Koehler, E., Langkau, K. and Skutella, M. (2002) Time-expanded graphs for flow-dependent transit times. R. Moehring and R. Raman (Eds.): ESA 2002, LNCS 2461, Springer-Verlag Berlin Heidelberg, 599-611.

Kotnyek, B. (2004) An annotated overview of dynamic network flows. INRIA, Sophia Antipolis, 1-28.

Kotusevski, G and Hawick, K.A. (2009) A review of traffic simulation software. Technical report CSTN-095, Computer Science, Institute of Information and Mathematical Sciences, Massy University, Albany, Auckland, New Zealand.

Lu, Q., Huang, Y. And Shekhar, S. (2003) Evacuation planning: a capacity constrained routing approach. Proceedings of the First NSF/NIJ Symposium on Intelligence and Security Informatics, 111-125.

$\mathrm{Lu}$, Q., George, B. and Shekhar, S. (2006) Capacity constrained routing algorithm for evacuation planning. Technical Report, Department of Computer Science and Engineering, University of Minnesota, 06-017.

Lu, Q., George, B. and Shekhar, S. (2005a) Capacity constrained routing algorithm for evacuation planning: a summary of results. Proceedings of the 9th International Symposium on Spatial and Temporal Databases (SSTD), 291-307.

Lv, N., Yan, X., Xu, K., and Wu, C. (2010) Bi-level programming based contraflow optimization for evacuation events. Kybernetes, 39:1227-1234.

Megiddo, N. (1974) Optimal flows in networks with multiple sources and sinks. Mathematical Programming,7:97-107.

Miller-Hooks, E. and Patterson, S.S. (2004) On solving quickest time problems in time-dependent. dynamic networks. Journal of Mathematical Modelling and Algorithms, 3:39-71.

Min, M. and Lee, J. (2013) Maximum throughput flow-based contraflow evacuation routing algorithm. Third International Workshop on Pervasive Networks for Emergency Management San Diego, 511--516.

Minieka, E. (1973) Maximal, lexicographic, and dynamic network flows. Operations Research, 21:517-527.

Moriarty, K., Ni, D. and Collura, J. (2007) Modeling traffic flow under emergency evacuation situations: Current practice and future directions. 86th Transportation Research Board Annual Meeting, Washington, D.C. Paper 07-0745.

Orlin, J.B. (1984) Minimum convex cost dynamic network flows. Mathematics of Operations Research 9:190207.

Pardalos, P.M. and Arulselvan, A. (2009) Multimodal solutions for large scale evacuations. Center for Multi- 
modal Solutions for Congestion Mitigation, Department of Industrial and System Engineering, University of Florida.

Pel, A.J., Bliemer, M.C.J. and Hoogendoorn, S.P. (2012) A review on travel behaviour modelling in dynamic traffic simulation models for evacuations. Transportation, 39:97-123.

Philpott, A.B. (1990) Continuous time flows in networks. Mathematical Methods of Operations Research, 15:640-661.

Pidd, M., de Silva, F. and Eglese, R. (1993) A configurable evacuation management and planning System. Proceedings of the Winter Simulation Conference, 1319-1323.

Pullan, M.C. (1993) An algorithm for a class of continuous linear programs. SIAM Journal on Control and Optimization, 31:1558-1577.

Pyakurel, U., Hamacher, H.W. and Dhamala T.N. (2014) Generalized maximum dynamic contraflow on lossy network. International Journal of Operations Research Nepal, 3:27-44.

Pyakurel, U., Goerigk, M., Dhamala T.N. and Hamacher, H.W. (2014) Transit dependent evacuation planning for Kathmandu valley: A case study. Technical Report (153), Department of Mathematics, TU Kaiserslautern, Germany.

Pyakurel, U., Goerigk, M., Dhamala, T. N. and Hamacher, H. W. (2015) Transit dependent evacuation planning for Kathmandu valley: A case study. International Journal of Operations Research Nepal, 5: 49-73.

Pyakurel, U. and Dhamala, T.N. (2014a). Earliest arrival contraflow model for evacuation planning. Neural, Parallel, and Scientific Computations [CNLS-2013], 22: 441-450.

Pyakurel, U. and Dhamala, T. N. (2015) Models and algorithms on contraflow evacuation planning network problems. International Journal of Operations Research, 12:36--46.

Pyakurel, U. and Dhamala, T.N. (2015a) Evacuation planning by earliest arrival contraflow. Journal of Industrial and Management Optimization (accepted).

Pyakurel, U. and Dhamala, T. N. (2015b) Continuous time dynamic contraflow models and Algorithms. Advance in Operations Research (under review).

Radwan, A., Hoebeika, A. and Sivasailam, D. (1985) A computer simulation model for rural network evacuation under natural disaster. Institute of Transportation Engineers Journal, 55:25-30.

Rebennack, S., Arulselvan, A., Elefteriadou, L. and Pardalos, P.M. (2010) Complexity analysis for maximum flow problems with arc reversals. Journal of Combinatorial Optimization, 19:200-216.

Ruzika, S., Sperber, H. and Steiner, M. (2011) Earliest arrival flows on series- parallel graphs. Networks, 10:169-173.

Sheffi, Y., Mahmassani, H. and Powell, W. (1980) NETVAC: A transportation network evacuation model. Center for Transportaion Studies, MIT.

Sisiopiku, V., Jones S., Sullivan A., Pathakar, S and Tang, X. (2004), Regional traffic simulation femergency preparedness. Technical Report, University Transportation Center for Alabama, the university of Alabama, the University of Alabama in Birmingham, and the University of Alabama in Huntsville.

Tagliaferri, A.P. (2005) Use and comparison of traffic simulation models in the analysis of emergency evacuation conditions. Ph.D. thesis, Department of Civil Engineering, North Carolina State University.

Wang, J. W., Ip, W. H. and Zhang, W. J. (2010) An integrated road construction and resource planning approach to the evacuation of victims from single source to multiple destinations. Transactions on Intelligent Transportation Systems, 11:277-289.

Wang, J. W., Wang, H. F., Zhang, W. J., Ip, W. H. and Furuta, K. (2013) Evacuation planning based on the contraflow technique with consideration of evacuation priorities and traffic setup time. IEEE Transactions on Intelligent Transportation Systems, 14: 480-485.

Wilkinson, W.L. (1971) An algorithm for universal maximal dynamic flows in a network. Operations Research, 19:1602-1612.

Williams, B., Tagliaferri, A., Meinhold, S., Hummer, J. and Rouphail, N. (2007) Simulation and analysis of freeway lane reversal for coastal hurricane evacuation. Journal of Urban Planning and Development, 133: 61-72.

Xie, C. and Turnquist, M. A. (2011) Lane-based evacuation network optimization: An integrated Lagrangian relaxation and tabu search approach. Transportation Research Part C, 19:40-63.

Xie, C. Lin, D .Y. and Waller, S.T. (2010) A dynamic evacuation network optimization problem with lane reversal and crossing elimination strategies. Transportation Research Part E, 46:295-316.

Yusoff, M., Ariffin, J. and Mohamed, A. (2008) Optimization approaches for macroscopic emergency evacuation planning: a survey. Information Technology, ITSim, International Symposium, IEEE, 26-28 August, 1--7.

Zhou, X., George, B., Kim, S., Wolff, J.M.R., Lu, Q. and Shekhar, S. (2010) Evacuation planning, a spatial network database approach. Bulletin of the IEEE Computer Society Technical Committee on Data Engineering, 1-6. 